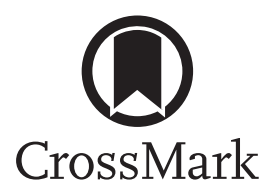

\title{
Closing the gap in surveillance of tuberculosis and HIV co-infection: a European perspective on the need for clinician-public health alliances
}

\author{
Marieke J. van der Werf (10) ${ }^{1}$, Giovanni Sotgiu (10 ${ }^{2}$ and Masoud Dara $\mathbb{1}^{3}$ \\ Affiliations: ${ }^{1}$ European Centre for Disease Prevention and Control, Stockholm, Sweden. ${ }^{2}$ Clinical Epidemiology \\ and Medical Statistics Unit, Dept of Biomedical Sciences, University of Sassari, Sassari, Italy. ${ }^{3}$ WHO Regional \\ Office for Europe, Copenhagen, Denmark. \\ Correspondence: M.J. van der Werf, European Centre for Disease Prevention and Control, Tomtebodavägen \\ 11A, Solna 17165, Stockholm, Sweden. E-mail: Marieke.vanderWerfdecdc.europa.eu
}

@ERSpublications

Clinicians and public health professionals need quality information on TB-HIV co-infection and should join forces http://ow.ly/Lacb30fKA3T

Cite this article as: van der Werf MJ, Sotgiu G, Dara M. Closing the gap in surveillance of tuberculosis and HIV co-infection: a European perspective on the need for clinician-public health alliances. Eur Respir J 2017; 50: 1701758 [https://doi.org/10.1183/13993003.01758-2017].

Surveillance of tuberculosis (TB) is essential for monitoring progress towards achieving the targets set in the End TB strategy [1] and to inform stakeholders whether prevention and control efforts are effective. In the World Health Organization (WHO) European Region, TB surveillance has a long history and is, overall, complete and accurate; however, surveillance of TB-HIV co-infection is hampered by deficiencies [2]. Obtaining high quality surveillance information requires the concerted action of professionals working in the clinical and public health sectors. In addition, TB-HIV co-infection surveillance requires close collaboration between clinicians working with $\mathrm{TB}$ and with HIV-infected patients. In this editorial we elaborate on the rational of why surveillance of TB-HIV co-infection is important both for clinical and public health purposes, how clinicians can contribute to high quality surveillance and how they would benefit from sound surveillance.

Global TB control was guided successfully by the WHO directly observed treatment, short-course (DOTS) strategy in the 1990s [3]. However, the emergence and spread of two TB-related determinants at the end of the 20th century, namely TB-HIV co-infection and drug-resistant TB, dramatically altered the scenario. These epidemics severely undermined past achievements, and, in some settings, reversed the impact of national and international public health interventions to reduce morbidity and mortality. The WHO Stop TB strategy, released in 2006, was aimed at addressing these new epidemiological threats [4]. This well-designed public health approach has succeeded, especially in settings where it was fully implemented and combined with permanent political commitment. The introduction of standards of care for TB has also contributed to improved diagnosis, treatment and prevention of TB in high and low TB incidence countries [5]. However, settings which faced political, socioeconomic and health system challenges did not fully experience the positive effects [6].

TB-HIV co-infection has mainly hit low-income countries where the availability of effective anti-HIV drugs is poor. Following regulatory approval, high-income countries, including the European Union (EU)

Received: Aug 282017 | Accepted after revision: Sept 072017

Conflict of interest: None declared.

Copyright OERS 2017 
and European Economic Area (EEA) countries, have had the chance to introduce HIV treatment with protease inhibitors in a timely manner, followed by the introduction of non-nucleoside reverse transcriptase inhibitors and, more recently, new drug categories (e.g. integrase inhibitors). The breakthrough discovery of highly active antiretroviral therapies and their immediate and large-scale availability in most of the developed countries has favoured the reduction of the size of the immunocompromised population, decreasing their lifetime probability of developing active TB. However, despite these therapeutic advances, the heterogeneous global distribution of antiretrovirals and the fact that national strategies addressing TB-HIV co-infection are not universally available or universally implemented has limited the potential impact, as in shown by the dramatic estimates published in 2016 by the WHO (400000 TB-HIV deaths and 1.2 million TB-HIV incident cases out of 10.4 million TB patients (11.5\%) in 2015) [3].

In the WHO European Region, the percentage of TB cases co-infected with HIV is increasing, due to both an overall decline in the number of non-HIV-infected cases and an increase in the number of co-infected cases [2]. In 2015, based on HIV prevalence data among notified TB patients, 16380 TB patients were reported as co-infected with HIV (i.e. 9\% of those with a known HIV status) [2]. This proportion varied from $0 \%$ in Bosnia Herzegovina, Iceland, the Former Yugoslav Republic of Macedonia, Slovakia and Slovenia, to $22.3 \%$ in Ukraine. In the EU, $16 \%$ of new AIDS cases presented with TB as an AIDS-defining illness in 2015 [7]. These data show that there is a considerable TB-HIV co-infection burden in the region and actions to prevent and control this are well defined [8-10] (Box 1). However, it remains the case that TB treatment outcomes are worse in TB-HIV co-infected patients compared to non-co-infected patients, suggesting that there is a need for targeted action [11].

Effective and efficient interventions targeting TB-HIV co-infection need to be guided and monitored by robust data. These data can be provided by disease surveillance, a public health activity that focusses on continuous, systematic collection of health-related data that are analysed, interpreted and thereafter used to guide action. The WHO Regional Office for Europe and the European Centre for Disease Prevention and Control (ECDC) collaborate and jointly coordinate TB surveillance in Europe. Data from national surveillance systems are collected through annual data calls, analysed and published to guide regional and country level actions. Next to annual reports with an in-depth analysis of the surveillance data [2], information is made accessible through websites, e.g. WHO's Global Tuberculosis Database (www.who.int/ tb/country/data/download/en/) and the ECDC surveillance atlas of infectious diseases (http://atlas.ecdc. europa.eu/public/index.aspx?Instance=GeneralAtlas).

To make surveillance data useful for guiding action they need to be of sufficient quality, which includes being complete, timely, reliable and valid. Even though it is mandatory to report TB cases in most countries [12, 13], the surveillance data on TB-HIV co-infection available in Europe lack these qualifications, with completeness being especially poor. Of the 53 countries in the WHO European region, plus Liechtenstein, 17 countries (31\%) did not report the HIV status of TB patients, of which 12 are EU and EEA countries. Of the 37 countries that did provide surveillance data on TB-HIV co-infection, information was available for $88 \%$ of TB patients in a range of 2 to $100 \%$ [2] (figure 1). In the 19 EU/EEA countries reporting on HIV status, information was available for $68 \%$ of TB patients.

Countries that do not collect surveillance information on TB-HIV co-infection might use other approaches to estimate the HIV-prevalence among TB patients. For example, Germany has evaluated available anonymous HIV and TB data sources and used the demographic characteristics of HIV/TB patients to estimate the proportion of TB patients that are co-infected [14]. Although valuable, information from such studies is not as useful as information that is readily available from surveillance systems, which allows insight to be gained into the real situation.

Box 1 World Health Organization (WHO) recommended collaborative tuberculosis (TB)/HIV activities with relevance for clinicians [10]

A. Reduce the burden of TB in people living with HIV and initiate early antiretroviral therapy

A.1. Intensify TB case-finding and ensure high quality antituberculosis treatment

A.2. Initiate TB prevention with isoniazid preventive therapy and early antiretroviral therapy

A.3. Ensure control of TB infection in health-care facilities and congregate settings

B. Reduce the burden of HIV in patients with presumptive and diagnosed TB

B.1. Provide HIV testing and counselling to patients with presumptive and diagnosed TB

B.2. Provide HIV prevention interventions for patients with presumptive and diagnosed TB

B.3. Provide co-trimoxazole preventive therapy for TB patients living with HIV

B.4. Ensure HIV prevention interventions, treatment and care for TB patients living with HIV

B.5. Provide antiretroviral therapy for TB patients living with HIV 


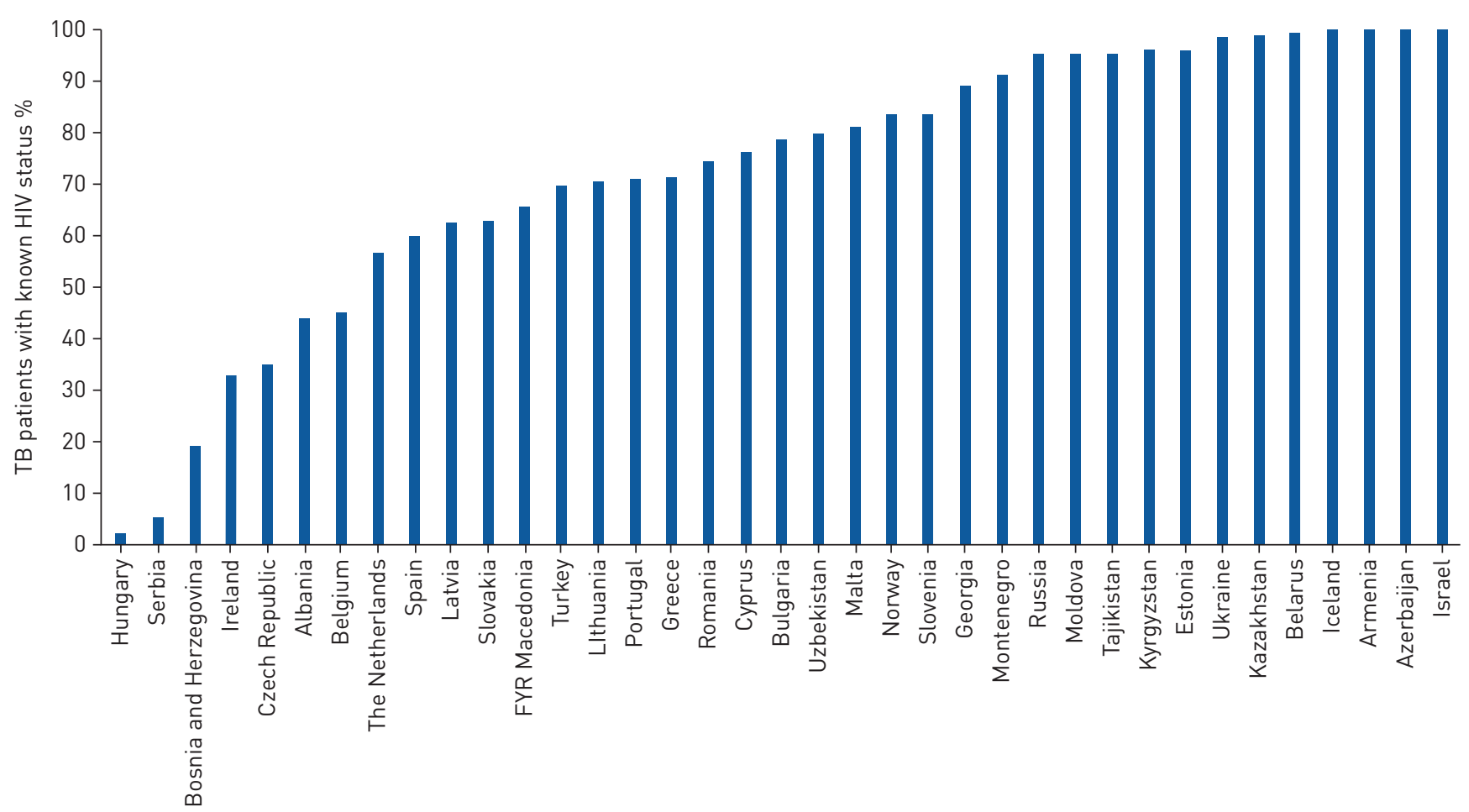

FIGURE 1 Percentage of tuberculosis (TB) patients with known HIV status in European countries that report on HIV status, 2015. Countries that did not report on HIV status included: Andorra, Austria, Croatia, Denmark, Finland, France, Germany, Italy, Liechtenstein, Luxembourg, Monaco, Poland, San Marino, Sweden, Switzerland, Turkmenistan and the United Kingdom.

Even if HIV status is reported for all TB patients notified through the TB surveillance system, surveillance information might still not be optimal since currently not all TB patients and TB-HIV co-infected patients are notified to the TB surveillance system [15-17]. A study in the Netherlands linking the national disease databases on TB and HIV showed that of 932 TB-HIV co-infected patients, only 293 (31.4\%) were registered in both the TB and the HIV registers, indicating that both surveillance registers are incomplete [18].

The main reported barriers to surveillance of TB-HIV co-infection were legal issues related to patient confidentiality and the anonymous testing and reporting of HIV infection [19]. However, these barriers are not universal since many countries do report on the HIV status of TB patients. Also, international guidelines underline that surveillance of HIV should be conducted among TB patients in order to inform programme planning and implementation [10].

It is within the mandate of the public health sector to develop systems and guidance that allow for the notification of TB-HIV co-infection. When these systems are in place, clinicians have an important role in, first of all, providing HIV testing and counselling for TB patients and, thereafter, in reporting HIV status to the surveillance system while respecting patients' confidentiality. Doing this will provide important information for the prevention and control of TB and especially of TB-HIV co-infection. Likewise, detection of TB among people living with HIV should also be recorded and reported. The information derived from such comprehensive systems will provide clinicians with an overview of their co-infected patients and their treatment outcomes and allow comparison of results at the local, national, or supranational level. This information can then be used to guide decision making on diagnostic and treatment processes.

A new alliance among all stakeholders (pulmonologists, HIV and infectious diseases specialists, patients' associations and public health officers) is crucial if we are to detect and report on all cases with TB-HIV co-infection, especially among the key populations. A more comprehensive approach, including cooperation among primary care physicians, clinician specialists and social workers, would further improve completeness, accuracy and timeliness of TB/HIV co-infection data. Clinicians can provide accurate data and information from a quality surveillance system can help in aligning treatment and case finding approaches. Currently, the WHO, the European Respiratory Society and other partners are working on strengthening active drug adverse effect monitoring, which can provide further evidence in terms of patient safety. In addition, national plans based on the early and active detection of TB-HIV 
infected persons are essential. These national health plans should include relevant national and international partners in order to work in true partnership to address TB-HIV co-infection.

\section{References}

1 Uplekar M, Weil D, Lonnroth K, et al. WHO's new End TB strategy. Lancet 2015; 385: 1799-1801.

2 European Centre for Disease Prevention and Control/WHO Regional Office for Europe. Tuberculosis surveillance and monitoring in Europe 2017. Stockholm, ECDC, 2017. https://ecdc.europa.eu/sites/portal/files/media/en/ publications/Publications/ecdc-tuberculosis-surveillance-monitoring-Europe-2017.pdf Date last accessed: October 09, 2017.

3 Global tuberculosis report 2016. Geneva, World Health Organization, 2016. http://apps.who.int/iris/bitstream/ 10665/250441/1/9789241565394-eng.pdf?ua=1 Date last accessed: October 09, 2017.

4 World Health Organization/Stop TB Partnership. The stop TB strategy: building on and enhancing DOTS to meet the TB-related millennium development goals. Geneva, World Health Organization, 2006. http://apps.who.int/iris/ bitstream/10665/69241/1/WHO_HTM_STB_2006.368_eng.pdf Date last accessed: October 09, 2017.

5 International standards for tuberculosis care. 3rd Edn. The Hague, TB CARE I, 2014. www.who.int/tb/ publications/ISTC_3rdEd.pdf Date last accessed: October 09, 2017.

6 Dirlikov E, Raviglione M, Scano F. Global tuberculosis control: toward the 2015 targets and beyond. Ann Intern Med 2015; 163: 52-58.

7 European Centre for Disease Prevention and Control/WHO Regional Office for Europe. HIV/AIDS surveillance in Europe 2015. Stockholm, ECDC, 2016. https://ecdc.europa.eu/sites/portal/files/media/en/publications/Publications/ HIV-AIDS-surveillance-Europe-2015.pdf Date last accessed: October 09, 2017.

8 Migliori GB, Zellweger JP, Abubakar I, et al. European Union standards for tuberculosis care. Eur Respir J 2012; 39: 807-819.

9 van der Werf MJ, Kodmon C, Zucs P, et al. Tuberculosis and HIV coinfection in Europe: looking at one reality from two angles. AIDS 2016; 30: 2845-2853.

10 WHO policy on collaborative TB/HIV activities: guidelines for national programmes and other stakeholders. Geneva, World Health Organization, 2012. http://apps.who.int/iris/bitstream/10665/44789/1/9789241503006_eng. pdf?ua $=1$ \&ua $=1$ Date last accessed: October 09, 2017.

11 Karo B, Krause G, Hollo V, et al. Impact of HIV infection on treatment outcome of tuberculosis in Europe. AIDS 2016; 30: 1089-1098.

12 Mor Z, Migliori GB, Althomsons SP, et al. Comparison of tuberculosis surveillance systems in low-incidence industrialised countries. Eur Respir J 2008; 32: 1616-1624.

13 Uplekar M, Atre S, Wells WA, et al. Mandatory tuberculosis case notification in high tuberculosis-incidence countries: policy and practice. Eur Respir J 2016; 48: 1571-1581.

14 Fiebig L, Kollan C, Hauer B, et al. HIV-prevalence in tuberculosis patients in Germany, 2002-2009: an estimation based on HIV and tuberculosis surveillance data. PLoS One 2012; 7: e49111.

15 Baussano I, Bugiani M, Gregori D, et al. Undetected burden of tuberculosis in a low-prevalence area. Int J Tuberc Lung Dis 2006; 10: 415-421.

16 Cojocaru C, van Hest NA, Mihaescu T, et al. Completeness of notification of adult tuberculosis in Iasi County, Romania: a capture-recapture analysis. Int J Tuberc Lung Dis 2009; 13: 1094-1099.

17 van Hest NA, Story A, Grant AD, et al. Record-linkage and capture-recapture analysis to estimate the incidence and completeness of reporting of tuberculosis in England 1999-2002. Epidemiol Infect 2008; 136: 1606-1616.

18 van Leth F, Evenblij K, Wit F, et al. TB-HIV co-infection in the Netherlands: estimating prevalence and under-reporting in national registration databases using a capture-recapture analysis. J Epidemiol Community Health 2016; 70: 556-560.

19 Kruijshaar ME, Pimpin L, Abubakar I, et al. The burden of TB-HIV in the EU: how much do we know? A survey of surveillance practices and results. Eur Respir J 2011; 38: 1374-1381. 